\title{
The Similarity Hypothesis and New Analytical Support on the Estimation of Horizontal Infiltration into Sand
}

\author{
Celso L. Prevedello ${ }^{1}$ and Jocely M. T. Loyola ${ }^{2}$ \\ ${ }^{1}$ Soils and Agricultural Engineering Department, Federal University of Paraná Rua dos Funcionários 1540, \\ 80030-035 Curitiba, PR, Brazil \\ ${ }^{2}$ Civil Construction Department, Federal University of Paraná, 81531-990 Curitiba, PR, Brazil \\ Correspondence should be addressed to Celso L. Prevedello, clpreve@gmail.com
}

Received 23 December 2009; Accepted 20 November 2010

Academic Editor: Artemi Cerda

Copyright ( 92010 C. L. Prevedello and J. M. T. Loyola. This is an open access article distributed under the Creative Commons Attribution License, which permits unrestricted use, distribution, and reproduction in any medium, provided the original work is properly cited.

\begin{abstract}
A method based on a specific power-law relationship between the hydraulic head and the Boltzmann variable, presented using a similarity hypothesis, was recently generalized to a range of powers to satisfy the Bruce and Klute equation exactly. Here, considerations are presented on the proposed similarity assumption, and new analytical support is given to estimate the water density flux into and inside the soil, based on the concept of sorptivity and on Buckingham-Darcy's law. Results show that the new analytical solution satisfies both theories in the calculation of water density fluxes and is in agreement with experimental results of water infiltrating horizontally into sand. However, the utility of this analysis still needs to be verified for a variety of different textured soils having a diverse range of initial soil water contents.
\end{abstract}

\section{Introduction}

Based on physical laws of similarity applied to the rate of work required for water to wet and move through a soil, Prevedello et al. [1] presented an analytic solution of a Boltzmann transformed equation of continuity for horizontal infiltration which is derived without invoking the concept or use of the soil water diffusivity function. The derivation assumes that a similarity exists between the shapes of the soil water retention function $\theta(h)$ and the Boltzmann transformation $\lambda^{2}(\theta)$, and the solution successfully described soil water content profiles experimentally measured for different infiltration times into a homogeneous sand. More recently, an extension of this theory generalized the solution to a range of powers to include the saturated zone [2], to satisfy the Bruce and Klute equation exactly.

With a similar assumption, but not exactly as expressed by Prevedello et al. [1], Prevedello et al. [3] obtained a new analytic solution of the Richards equation for the infiltration into the same sand that holds for all infiltration times from zero to infinity, including vertical directions without making use of empirical constants. In this case, the derivation assumed that a similarity exists between the soil water retention function $\theta(h)$ and the soil water content distribution $\theta(z)$ within the soil profile during infiltration.

Although the similarity assumptions used by $[1,3]$ seem different, we now show that the mathematical development of both leads to the same equation for the description of the soil water content profiles for horizontal infiltration. We also present an analysis to obtain analytical equations to estimate the water density flow into and inside the soil, according to the sorptivity concept and the Buckingham-Darcy law. These analytical equations are compared with experimental results of horizontal infiltration into the same homogeneous marine sand.

\section{Theoretical Considerations}

2.1. Extended Similarity Hypothesis $\lambda^{N}$. Two identical solutions for the horizontal water infiltration into a soil were presented by $[1,3]$, coincidently (39) in both papers, but using two apparently different initial postulates. The explanation for that is given by a generalization of the similarity theory proposed in [1]. A generalization of the similarity 
between the Boltzmann transformation $\lambda^{2}(\theta)$ and the soil water retention function $\theta(h)$ can be written as follows:

$$
\lambda^{N} \frac{d \theta}{d \lambda^{N}}=h \frac{d \theta}{d h}
$$

in which $N$ is a positive integer, $\theta$ the soil water content $\left(\mathrm{m}^{3} \mathrm{~m}^{-3}\right)$, and $h$ the matric water potential head $(m)$. With (1) being the fundamental hypothetical equation, it follows that

$$
\frac{d \lambda^{N}}{\lambda^{N}}=\frac{d h}{h}
$$

or

$$
\ln \lambda^{N}=\ln h+\ln C_{N},
$$

and introducing the horizontal position coordinate $x(m)$ and the time (s), we have

$$
\lambda=x t_{m}^{-1 / 2}=C_{N}^{1 / N} h^{1 / N}
$$

note that $t_{m}=t_{\text {model }}=\left(t_{\text {measured }}\right) / N$.

When $t_{m}=1$,

$$
\lambda=x=C_{N}^{1 / N} h^{1 / N} .
$$

From (2) it follows that

$$
\begin{gathered}
\frac{d h}{d x}=\frac{N h}{x}, \\
\frac{\partial h}{\partial x}=\frac{N h_{x=x}}{x} .
\end{gathered}
$$

Substituting (7) into the Buckingham-Darcy equation written in the $x$-direction in terms of the soil hydraulic conductivity $K$ as a function of $\theta$, we have

$$
q_{m}=-K(\theta)\left\lfloor\frac{N h_{x=x}}{x}\right\rfloor
$$

where the subscript $m$ refers to the model hypothesis of (4). Knowing (8), changing the variables so that the dependent variable becomes the distance $x$, and remembering that $x=$ $f(\theta, t)=$ constant because the partial derivative of $\theta$ is taken with respect to $t$, we have

$$
\frac{\partial x}{\partial t_{m}}=-\frac{\partial}{\partial \theta}\left\lfloor K(\theta) \frac{N h_{x=x}}{x}\right\rfloor,
$$

which leads to

$$
\frac{\partial x}{\partial t_{m}}=\frac{N K(\theta) h_{x=x}}{x^{2}} \frac{\partial x}{\partial \theta}-\frac{N K(\theta)}{x} \frac{\partial h_{x=x}}{\partial \theta}-\frac{N h_{x=x}}{x} \frac{\partial K(\theta)}{\partial \theta} .
$$

For a fixed time, based on (7), and multiplying by $\Delta \theta \cdot \Delta t_{m}$, we have

$x \frac{\partial x}{\partial t} \Delta t_{m} \Delta \theta=(1-N) K(\theta) \frac{\partial h}{\partial \theta} \Delta \theta \Delta t_{m}-N h_{x=x} \frac{\partial K(\theta)}{\partial \theta} \Delta \theta \Delta t_{m}$ or

$$
x \Delta x \Delta \theta=(1-N) K(\theta) \Delta h \Delta t_{m}-N h_{x=x} \Delta K \Delta t_{m} .
$$

Holding $h$ constant and integrating, the above equation becomes

$$
\int_{0}^{x} x d x \int_{\theta_{i}}^{\theta_{0}} d \theta=-N h_{x=x} \int_{K_{i}}^{K_{0}} d \theta \int_{0}^{t_{m}} d t
$$

Evaluating at $t_{m}=1$ when $x=\lambda$,

$$
\begin{gathered}
\int_{0}^{\lambda} \xi d \xi \int_{\theta_{i}}^{\theta_{0}} d \theta=-N h_{x=x} \int_{K_{i}}^{K_{0}} d K \int_{0}^{1} d t, \\
\frac{\lambda^{2}}{2}\left(\theta_{0}-\theta_{i}\right)=-N h_{x=x}\left(K_{0}-K_{i}\right), \\
\lambda^{2}=\frac{-2 N\left(K_{0}-K_{i}\right) h_{x=x}}{\left(\theta_{0}-\theta_{i}\right)} .
\end{gathered}
$$

Recalling (3) in the form of

$$
\lambda^{2}=\left(C_{N} h\right)^{2 / N}
$$

and equating the two above equations that define $\lambda^{2}$, we have

$$
\left(C_{N} h\right)^{2 / N}=\frac{-2 N\left(K_{0}-K_{i}\right) h_{x=x}}{\left(\theta_{0}-\theta_{i}\right)} .
$$

Returning to (4), we know that

$$
x=\left(C_{N} h\right)^{1 / N} t_{m}^{1 / 2} .
$$

Substituting $C_{N}$ into the above equation, we obtain

$$
x=\left(\frac{-2\left(K_{0}-K_{i}\right) h_{x=x} N t_{m}}{\left(\theta_{0}-\theta_{i}\right)}\right)^{1 / 2} .
$$

Recalling that $N t_{\text {model }}=t_{\text {measured }}$, the solution is

$$
x=\sqrt{\frac{-2\left(K_{0}-K_{i}\right) h_{x=x} t_{\text {measured }}}{\left(\theta_{0}-\theta_{i}\right)}} .
$$

For the cases in which the soil is initially very dry (i.e., $K_{i}=0$ ), the above equation reduces to

$$
x=\sqrt{\frac{-2 K_{0} h_{x=x} t_{\text {measured }}}{\left(\theta_{0}-\theta_{i}\right)}}
$$

which is (39) presented in $[1,3]$.

From (5) we have

$$
\lambda=C_{N}^{1 / N} h^{1 / N} \quad \text { or } \quad \lambda^{N}=C_{N} h .
$$

According to (21), the generalized solution of $\lambda^{N}$ is

$$
\lambda^{N}=h^{N / 2-1}\left(\frac{-2 N\left(K_{0}-K_{i}\right)}{\left(\theta_{0}-\theta_{i}\right)}\right)^{N / 2} \cdot h .
$$

But the fundamental hypothesis that gave rise to (22) is

$$
\frac{d \lambda^{N}}{d h}=\frac{\lambda^{N}}{h} .
$$


The derivative of (22) with respect to $h$ is

$$
\frac{d \lambda^{N}}{d h}=\frac{h^{N / 2-1} N 2^{N / 2}\left(-N\left(K_{0}-K_{i}\right) /\left(\theta_{0}-\theta_{i}\right)\right)^{N / 2}}{2} .
$$

Dividing (22) by $h$,

$$
\frac{\lambda^{N}}{h}=h^{N / 2-1} 2^{N / 2}\left(\frac{-N\left(K_{0}-K_{i}\right)}{\left(\theta_{0}-\theta_{i}\right)}\right)^{N / 2}
$$

Equating (24) and (25), since both follow the postulate, the result is $N=2$. This shows that any value of $N$ different from 2 cannot be accepted in this theory of similarity, as originally postulated and confirmed in [1], since it is with the square root of the time that the advancement of the wetting front remains linear in the process of horizontal infiltration.

2.2. Explanation Notes. Considering $N=2\left[\right.$ or $\left.\lambda^{2}(\theta)\right]$ and since $x_{x=x}^{2} /\left(h_{x=x} t\right)=\beta=$ constant, [1, equation (19)], for the cases in which $h=0$ at $x=0$ and when the soil is initially very dry $\left(K_{i}=0\right)$, can be written as

$$
\frac{x_{x=x}^{2}}{h_{x=x} t}=\beta=-\frac{4 K_{0}}{\left(\theta_{0}-\theta_{i}\right)}+\frac{2 K_{0}}{\left(\theta_{0}-\theta_{i}\right)}=-\frac{2 K_{0}}{\left(\theta_{0}-\theta_{i}\right)} .
$$

Therefore, we know that

$$
\beta=-\frac{2 K_{0}}{\left(\theta_{0}-\theta_{i}\right)},
$$

and we can write (26) as follows:

$$
\frac{x_{x=x}}{\beta t}=\left(\frac{2 h_{x=x}}{x_{x=x}}-\frac{h_{x=x}}{x_{x=x}}\right)=\frac{h_{x=x}}{x_{x=x}}
$$

which shows that the gradient $\partial h / \partial x=2 h_{x=x} / x_{x=x}$ formulated as an initial postulate in [1] is simplified during the theoretical development to $h / x$. This statement can also be verified starting with (11). In this equation, holding $h$ constant and making $N t_{\text {model }}=t_{\text {measured }}$, we can write

$$
\Delta x \Delta \theta=-\frac{h_{x=x}}{x} \Delta K \Delta t_{\text {measured }}
$$

where we can see that the initially formulated gradient $N h / x$ becomes $h / x$. The integration of the equation above leads to (22) in $[1,3]$.

The solution obtained by [3] for horizontal infiltration case, although identical to that obtained by [1], presupposes a relation of the type $\lambda^{N}=C_{N} h$ with $N=1$, that is, $\partial h / \partial x=$ $h_{x=x} / x$ or $\ln \lambda=\ln h_{x=x}+C$ or $\lambda=C h$, and, therefore, it cannot be taken as formal as that presented in [1]. As explained above, this is due to the fact that independently of the chosen value of $N$ in the generalised similarity theory here introduced, the gradient $\partial h / \partial x=N h_{x=x} / x$ of (7), as initially postulated in [1], is always simplified during the theoretical development from $\left(N h_{x=x}\right) / x$ to $h_{x=x} / x$, so that the B-D equation is always converted to $q=-K(\theta)\left\lfloor h_{x=x} / x\right\rfloor$, and that, therefore, leads to a final solution that is identical to that obtained by [1].

In this way it is shown that the same soil water profile $x(\theta)$, expressed by $(22)$ in both papers $[1,3]$, can be obtained from both similarity approaches, that is, $\lambda^{2}(\theta)$ or $x(\theta)$ as images of the soil water retention curve $h(\theta)$. However, only $\lambda^{2}(\theta)$ as an image of $h(\theta)$ translates formally what was postulated into a solution, such as shown in [1] or in the extended similarity hypothesis $\lambda^{N}$ shown above, in which only $N=2$ carries out formally this postulate.

\subsection{The Similarity Hypothesis and New Analytical Support on} the Estimation of Horizontal Infiltration. Since Prevedello et al. [1] found a solution such that $\lambda^{2}$ is proportional to $h$, so that $d h / d x$ is proportional to $\lambda$, it follows that for $t>0$, from the definition of $\lambda$, at $x=0$ both $d h / d x$ and $\lambda$ are equal to zero. At a first glance, one could assume that to have a finite flux density $q$ at $x=0, K$ should be infinite at $\theta=\theta_{s}$. According to this interpretation, Barry et al. [2] introduced a nonzero air-entry value $h_{a}$ (e.g., Haverkamp et al. [4]), such that for $h<h_{a}, \theta<\theta_{O}$ the soil is unsaturated and saturated for $h \geq h_{a}, \theta=\theta_{O}$ and generalized the Prevedello et al. [1] solution to a range of powers. We here use the original interpretation of Prevedello et al. [1] to calculate the flux density $q$ at $x=0$ and at $x=x$, without running into the shortcomings mentioned above. From Phillip's analysis, we know that for the case of horizontal infiltration,

$q_{x=0}(t)=\frac{d i}{d t}=\frac{S}{2 t^{1 / 2}}=\left(-K(\theta) \frac{\partial h}{\partial x}\right)_{x=0}=\frac{1}{2 t^{1 / 2}} \int_{\theta_{i}}^{\theta_{O}} \lambda(\theta) d \theta$.

If in (30) we substitute $x$ by $\lambda t^{1 / 2}$ the Bruce and Klute equation or (1) of Barry et al. [2] are obtained. There are many analytical forms to prove the equality in (30). Here, our option was to obtain an analytical expression for both the members and to compare the results obtained for each one. According to the similarity assumption proposed by [1] we know that

$$
S=\sqrt{\frac{-2 K_{0}}{\theta_{0}-\theta_{i}}} \int_{\theta_{i}}^{\theta_{0}} \sqrt{h_{x=x}} d \theta .
$$

From van Genuchten we know that

$$
-h_{x=x}=\frac{1}{\alpha}\left(\left(\frac{\theta_{x=x}-\theta_{r}}{\theta_{0}-\theta_{r}}\right)^{-1 / m}-1\right)^{1 / n}
$$

so that (31) becomes

$$
S=\sqrt{\frac{2 K_{0}}{\alpha\left(\theta_{0}-\theta_{i}\right)}} \int_{\theta_{i}}^{\theta_{0}}\left(\left(\frac{\theta_{0}-\theta_{r}}{\theta_{x=x}-\theta_{r}}\right)^{1 / m}-1\right)^{1 / 2 n} d \theta,
$$

so that

$$
q_{x=0}(t)=\sqrt{\frac{K_{0}}{2 \alpha\left(\theta_{0}-\theta_{i}\right) t}} \int_{\theta_{i}}^{\theta_{0}}\left(\left(\frac{\theta_{0}-\theta_{r}}{\theta_{x=x}-\theta_{r}}\right)^{1 / m}-1\right)^{1 / 2 n} d \theta .
$$

The water flux density can also be evaluated through the Buckingham-Darcy law directly, using the Prevedello et al. $[1,3]$ similarity assumption:

$$
q_{x=0}=K_{o} \frac{\partial h}{\partial x}=K_{o} \frac{h_{x=x}}{x_{x=x}}=K_{o} \frac{h_{x=x}}{\sqrt{-2 K_{o} h_{x=x} t /\left(\theta_{o}-\theta_{i}\right)}} .
$$


But, according to the similarity assumption, each value of $h_{x=x}$ of the wetting profile is associated to its position value $x=x$. Therefore, $h_{x=x} / x$ does not represent directly the total or representative matric gradient at the wetting front. In this way, (35) should be interpreted as the water density flow that enters at $x=0$ in response to a "representative" gradient that occurs at the wetting front. Thus, if $\overline{h_{x=x}}$ is taken as the average matric potential of all the active potentials, then we can find analytically this representative matric potential, as well as the position where it acts at the wetting front, applying the similarity assumption. In this way, the previous equation can be written as

$$
q_{x=0}=K_{o} \frac{\partial h}{\partial x}=K_{o} \frac{\overline{h_{x=x}}}{\overline{x_{x=x}}}=K_{o} \frac{\overline{h_{x=x}}}{\sqrt{\left(-2 K_{o} \overline{h_{x=x}} t\right) /\left(\theta_{o}-\theta_{i}\right)}},
$$

where $\overline{h_{x=x}}$ is the representative potential active at the wetting front and $\overline{x_{x=x}}$ the position where this potential acts, defined by

$$
\sqrt{-\overline{h_{x=x}}}=\frac{\int_{\theta_{i}}^{\theta_{0}} \sqrt{-h_{x=x}} d \theta}{\int_{\theta_{i}}^{\theta_{0}} d \theta}
$$

or

$$
-\overline{h_{x=x}}=\left[\frac{1}{\left(\theta_{0}-\theta_{i}\right) \sqrt{\alpha}} \int_{\theta_{i}}^{\theta_{0}}\left(\left(\frac{\theta_{0}-\theta_{i}}{\theta_{x=x}-\theta_{i}}\right)^{1 / m}-1\right)^{1 / 2 n} d \theta\right]^{2} .
$$

Notice that this interpretation provides a constant value of $\bar{h}$ independent of time, and it follows that

$$
\bar{x}\left(\bar{h}_{x=x}, t\right)=\left(\frac{-2 \bar{h}_{x=x} K_{0}}{\left(\theta_{0}-\theta_{i}\right)}\right)^{1 / 2} t^{1 / 2} .
$$

From the soil surface, where the soil is saturated $(K=$ $K_{O}$ ), the wetting front advances as a response to an average matric potential $\bar{h}$, and with a force $\bar{h} / \bar{x}$ (average matric gradient),

$$
q_{x=0}(t)=\left(-K_{O} \frac{\bar{h}}{\bar{x}}\right)_{x=0}
$$

which should yield the same results as (34).

The analytical equation to estimate water density flow inside the soil can be determined simply by substituting $K_{O}$ for $K(\theta)$ or $K(h)$ into (40), because the average gradient remains fixed, but inside the soil we have the soil water content or matric potential for each position $x$ :

$$
q_{x=x}(t)=\left(-K\left(h_{x=x}\right) \frac{\bar{h}}{\bar{x}}\right)_{x=x} .
$$

\section{Material and Methods}

To validate experimentally the equations proposed above to quantify the water density into and inside the soil during
TABLe 1: Saturated hydraulic conductivity $K_{0}$ and parameters $a$, $n, m, q_{0}$, and $q_{r}$ of the van Genuchten [6] model for the wetting water retention curve of the marine sand and the coefficient of determination $r^{2}$.

\begin{tabular}{lcccccc}
\hline $\begin{array}{l}K_{0} \\
\left(\mathrm{~m} \cdot \mathrm{s}^{-1}\right)\end{array}$ & $\begin{array}{c}a \\
\left(\mathrm{~m}^{-1}\right)\end{array}$ & $n$ & $m$ & $\begin{array}{c}\theta_{0} \\
\left(\mathrm{~m}^{3} \cdot \mathrm{m}^{-3}\right)\end{array}$ & $\begin{array}{c}\theta_{r} \\
\left(\mathrm{~m}^{3} \cdot \mathrm{m}^{-3}\right)\end{array}$ & $r^{2}$ \\
\hline 0.0001583 & 4.1 & 17 & 0.9412 & 0.3870 & 0.0187 & 0.991 \\
\hline
\end{tabular}

the horizontal infiltration process for homogeneous sand, we used the same data presented in $[1,3]$.

The van Genuchten [6] model was used to describe the soil water retention curve

$$
h=-\frac{1}{\alpha}\left(\Theta^{-1 / m}-1\right)^{1 / n}, \quad \Theta=\frac{\theta-\theta_{r}}{\theta_{0}-\theta_{r}},
$$

where $\alpha, n, \theta_{0}, \theta_{r}$ (residual soil water content), and $m(m=$ $1-1 / n)$ are the classical parameters of the soil water retention curve. Using a nonlinear regression program as the one suggested by Boratto [5], (42) was fitted to experimental values to obtain the independent parameters $\alpha, \theta_{0}, \theta_{r}, n$, and $m$.

According to van Genuchten [6], introducing (42) into the Mualem [7] theory, the function $K(\Theta)$ is obtained as

$$
K(\Theta)=\Theta^{\frac{1}{2}}\left[1-\left(1-\Theta^{1 / m}\right)^{m}\right]^{2} .
$$

Data for the saturated hydraulic conductivity and for the parameters of the water retention curve for the marine sand are presented in Table 1 , as previously shown in $[1,3]$. With a coefficient of determination greater than 0.99 , the van Genuchten [6] model provides an excellent representation of the observed values.

\section{Results and Discussion}

4.1. The Water Density Flow into the Soil $(x=0)$, according to Philip's Concept of Sorptivity. From the boundary conditions and the soil parameters (Table 1 ) and assuming the initial soil water content equal to $\theta_{i}=0.0187 \mathrm{~m}^{3} \cdot \mathrm{m}^{-3}$, (34) reduces to

$$
q_{x=0}=\frac{S}{2 t^{1 / 2}}=\frac{20,7489184}{t^{1 / 2}} \mathrm{~mm} / \mathrm{min}
$$

which is shown in Figure 1 as a function of time, according to the analysis of Philip [8].

Measured soil water content profiles (Figure 5 of Prevedello et al. [1]) were integrated with respect to distance $x$ for times $t_{1}, t_{2}$, and $t_{3}$ equal to 15,60 , and $300 \mathrm{~min}$, resulting in 165,330 , and $732 \mathrm{~mm}$, respectively. We also know that the cumulative infiltration into the soil surface

is

$$
i(t)=\int_{\theta_{i}}^{\theta_{0}} x(\theta, t) d \theta=t^{1 / 2} \int_{\theta_{i}}^{\theta_{0}} \lambda(\theta) d \theta
$$

or

$$
i(t)=S t^{1 / 2}, \quad S=\int_{\theta_{i}}^{\theta_{0}} \lambda(\theta) d \theta,
$$




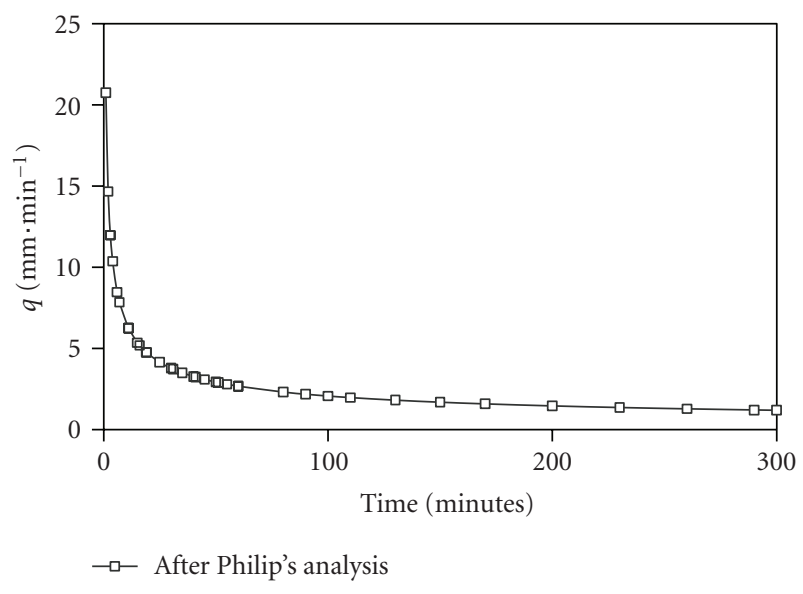

FIGURE 1: Water density flow into the soil $(x=0)$ as a function of the time for horizontal infiltration in homogeneous marine sand, according to (34) or (44).

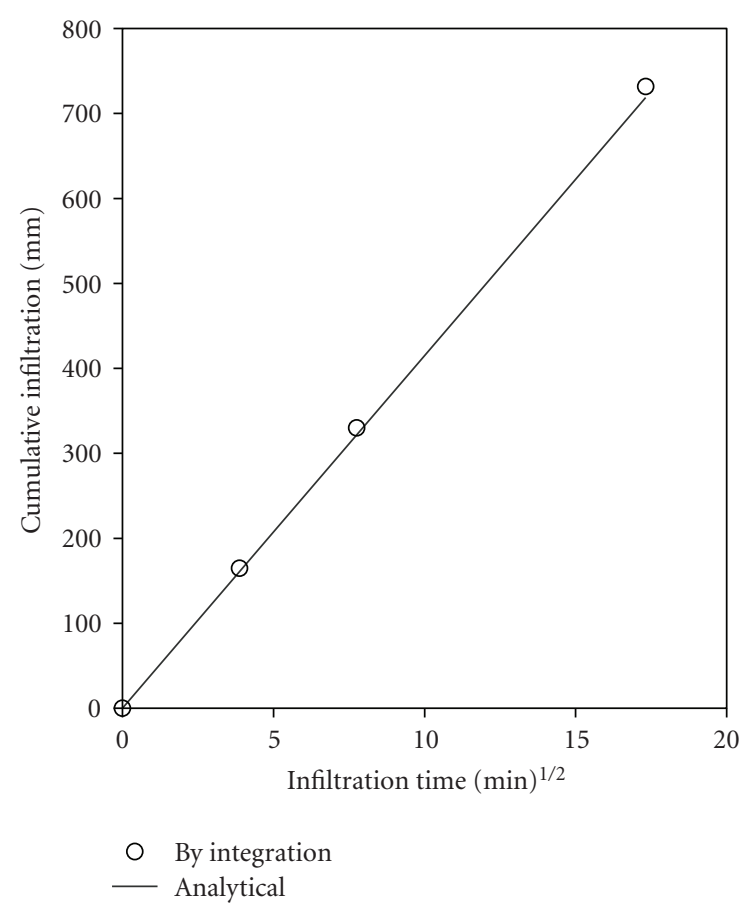

FIGURE 2: Cumulative water infiltration analytically estimated from (46) and observed data obtained integrating soil water profiles presented in [1].

where $S$ is the sorptivity.

In light of (46) and substituting the value $S=$ $41.4978368 \mathrm{~mm} \cdot(\mathrm{min})^{-1 / 2}$, we have $i(15)=160.7 \mathrm{~mm}$, $i(60)=321.44 \mathrm{~mm}$, and $i(300)=718.76 \mathrm{~mm}$, respectively, which are very close to those estimated by integration of experimental data and showed in Figure 2.

4.2. The Water Density Flow into the Soil $(x=0)$, According to Buckingham-Darcy Law. To verify the analytical validity of (40) to quantify the water density flow at $x=0,(38)$

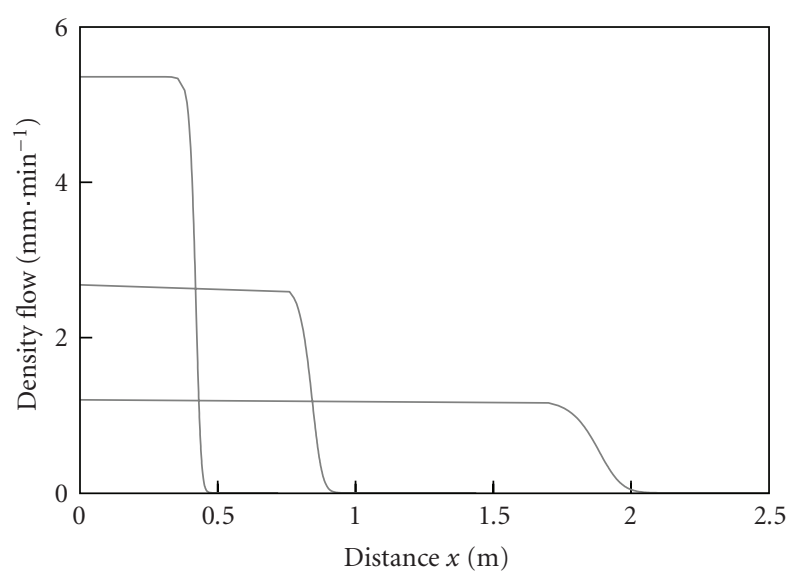

FIGURE 3: The water density flow as a function of $x$ inside the soil for times $t_{1}, t_{2}$, and $t_{3}$ equal to 15,60 , and $300 \mathrm{~min}$ (from up to down), after (49a) or (49b).

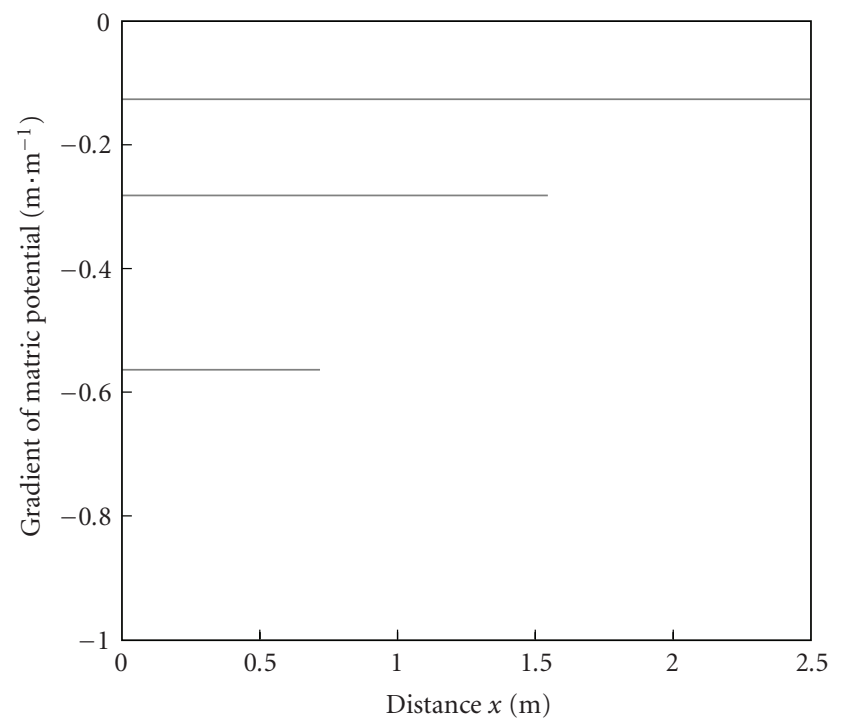

FIGURE 4: Matric gradient as a function of $x$ inside the soil for times $t_{1}, t_{2}$, and $t_{3}$ equal to 15,60 , and $300 \mathrm{~min}$ (from down to up), according to (50a) or (50b).

and (39) were evaluated, resulting, respectively, in $\bar{h}=$ $-0.246090924 \mathrm{~m}$ and $\bar{x}\left(\bar{h}_{x=x}, t\right)=0.112674007 \cdot t^{1 / 2}$. These results show that the similarity theory proposed by [1], here represented by (36) or (40), does not imply in a water flux density zero at $x=0$. Substituting these values and $K_{O}=$ $0.0095 \mathrm{~m} / \mathrm{min}$ into (40), we obtain

$$
q_{x=0}=\frac{20.7489184}{t^{1 / 2}} \mathrm{~mm} / \mathrm{min}
$$

which is exactly the same value obtained in the calculation through Philip's concept for sorptivity (44) and what therefore also adjusts very well with the experimental results showed in the Figure 2.

Another apparently analogous way to obtain the results of (47) above is to consider the derivative $\partial h / \partial x$ from (20), 
that is, $\partial h / \partial x=-\left(\left(\left(\theta_{0}-\theta_{i}\right) \cdot x\right) / K_{0} t\right)$. But according to the similarity theory proposed in $[1,3]$, the value of $h_{x=x}$ is a constant for each position $x$ at which it is only dependent on the time $t$ of advancement, and the derivative of a constant is null. The same restriction is applicable to the Green and Ampt theory [9] when we want to obtain the matric gradient through derivative of $h_{\mathrm{wf}}$ (the pressure head at the wetting front) with respect to $x$ because $h_{\mathrm{wf}}$ is always a constant independent of $x$. So, the derivative of $h$ with respect to $x$ cannot be used here, except if associated to the concept of the similarity, and as exposed previously in item b above, we know that the matric gradient $\partial h / \partial x=2 h_{x=x} / x_{x=x}$ is reduced to $h_{x=x} / x_{x=x}$, and therefore we can also write (40) as

$$
q_{x=0}(t)=\left(-K_{O} \frac{\bar{h}}{\bar{x}}\right)_{x=0}=\left(-\frac{\left(\theta_{0}-\theta_{i}\right) \cdot \bar{x}}{2 t}\right) .
$$

Comparing (30) with (48), we know that $K(\theta)=0$ for $h<\bar{h}, K(\theta)=K_{o}$ for $\bar{h}<h \leq 0$, and $h_{x=x}=\bar{h}$ for $h \leq 0$. It is now readily apparent from the above analysis that the distribution of $K(\theta)$ was substituted by the constant $K_{o}$ in (48) to determine the water flow density at $x=0$ in response to the force that occurs at distance $\vec{x}$ where $h_{x=x}=\bar{h}$. Inside of the soil the values of $K\left(\theta_{x=x}\right)$ or $K\left(h_{x=x}\right)$ are consistent with the values of $\theta\left(h_{x=x}\right)$ found from the values of $h_{x=x}$ in (39) of Prevedello et al. [1,3] because (39) provides the shape of the soil water content profile at the wetting profile while the average gradient $\bar{h} / \bar{x}$ remains active on the time.

Equation (48) with values of $\theta$ from Table 1 and $\bar{x}\left(\bar{h}_{x=x}, t\right)=0.112674007 \cdot t^{1 / 2}$ also yields values very close to the experimental results (Figure 2) and exactly the same results obtained in the calculation through Philip's concept for sorptivity (44).

4.3. The Density of Flow inside of the Soil, for Any Position and Time. Considering the boundary conditions of soil water content varying between 0.0187 and $0.387 \mathrm{~m}^{3} \cdot \mathrm{m}^{-3}$, to use (41) we first estimate the corresponding matric potentials for any $\theta$ stipulated between 0.0187 and $0.387 \mathrm{~m}^{3} \cdot \mathrm{m}^{-3}$, to obtain their respective positions from (20) and their respective $K\left(h_{x=x}\right)$ values from (43).

Through (41) we generate the water density flow for each position inside the soil, that is,

$$
q_{x=x}(t)=\left(-K\left(h_{x=x}\right) \frac{-0.246090924}{0.112674007}\right)_{x=x} \frac{1}{t^{1 / 2}} .
$$

Analogously as before in (48), the water density flow inside the soil can also be determined through the following:

$$
q_{x=x}(t)=\left(-K\left(h_{x=x}\right) \frac{\left(\theta_{0}-\theta_{i}\right) \bar{x}}{2 K_{0} t}\right)_{x=x}
$$

in which $\bar{x}\left(\bar{h}_{x=x}, t\right)=0.112674007 \cdot t^{1 / 2}$, and the values of $K_{0}$ and $\theta$ are showed in the Table 1.

Both (49a) and (49b) yield exactly the same values, and Figure 3 shows the water density flow estimated from those equations for each $x$ reached at 15,60 and 300 minutes of the horizontal infiltration into the homogeneous marine sand.
It can be noted that for $x=0$ the water density flow values coincide with those obtained from (44), (47), or (48), as it should be.

From the above, it follows that

$$
\frac{\partial h}{\partial x}=\frac{\bar{h}}{\bar{x}}=\left(\frac{-0.246090924}{0.112674007}\right) \frac{1}{t^{1 / 2}},
$$

$$
\begin{aligned}
& \text { or } \\
& \frac{\partial h}{\partial x}=\left(-\frac{\left(\theta_{0}-\theta_{i}\right) \bar{x}}{2 K_{0} t}\right)=\left(-\frac{\left(\theta_{0}-\theta_{i}\right) 0.112674007}{2 K_{0}}\right) \frac{1}{t^{1 / 2}},
\end{aligned}
$$

indicating that the gradient decreases with the time, shown in Figure 4 for times of 15, 60, and 300 minutes.

To verify how accurate these estimated results are, the same boundary value problem was solved numerically from Richard's equation without any similarity assumption, using the procedure of Philip [10] with appropriate initial and boundary conditions and using the measured soil water properties shown in Section 3 (calculations based on $\Delta \theta=$ $\left.0.0008 \mathrm{~m}^{3} \cdot \mathrm{m}^{-3}\right)$. Results showed that both water density flows at $x=0$ and inside the soil were very similar in the transmission zone and wetting front for each of the three chosen times of horizontal infiltration. However, the utility of this analysis still needs to be verified for a variety of different textured soils having a diverse range of initial soil water contents.

\section{Conclusion}

The new analytical supports to estimate the water density into and inside of the soil during horizontal infiltration agree with the experimental results, with the concept of sorptivity proposed by Philip [8] as well as the Buckingham-Darcy law, when a "representative" matric gradient is considered acting at the wetting front.

\section{Acknowledgment}

The authors acknowledge Donald R. Nielsen and Klaus Reichardt for their support and guidance in preparing this paper.

\section{References}

[1] C. L. Prevedello, J. M. T. Loyola, K. Reichardt, and D. R. Nielsen, "New analytic solution of Boltzmann transform for horizontal water infiltration into sand," Vadose Zone Journal, vol. 7, no. 4, pp. 1170-1177, 2008.

[2] D. A. Barry, J.-Y. Parlange, C. L. Prevedello, J. M.T. Loyola, K. Reichardt, and D. R. Nielsen, "Extension of a recent method for obtaining exact solutions of the Bruce and Klute equation," Vadose Zone Journal, vol. 9, no. 2, pp. 496-498, 2010.

[3] C. L. Prevedello, J. M. T. Loyola, K. Reichardt, and D. R. Nielsen, "New analytic solution related to the Richards, Philip, and Green-Ampt equations for infiltration," Vadose Zone Journal, vol. 8, no. 1, pp. 127-135, 2009. 
[4] R. Haverkamp, J. Y. Parlange, J. L. Starr, G. Schmitz, and C. Fuentes, "Infiltration under ponded conditions: 3. A predictive equation based on physical parameters," Soil Science, vol. 149, no. 5, pp. 292-300, 1990.

[5] F. Boratto, BASIC for Engineers and Scientists, Livros Técnicos e Científicos, Rio de Janeiro, Brazil, 2nd edition, 1984.

[6] M. TH. van Genuchten, "A closed-form equation for predicting the hydraulic conductivity of unsaturated soils," Soil Science Society of America Journal, vol. 44, no. 5, pp. 892-898, 1980.

[7] Y. Mualem, "A new model for predicting the hydraulic conductivity of unsaturated porous media," Water Resources Research, vol. 12, no. 3, pp. 513-522, 1976.

[8] J. R. Philip, "The theory of infiltration: 1. The infiltration equation and its solution," Soil Science, vol. 83, pp. 345-357, 1957.

[9] W. H. Green and G. A. Ampt, "Studies in soil physics. Part I. The flow of air and water through soils," The Journal of Agricultural Science, vol. 4, pp. 1-24, 1911.

[10] J. R. Philip, "Numerical solution of equations of the diffusion type with diffusivity concentration-dependent," Transactions of the Faraday Society, vol. 51, pp. 885-892, 1955. 

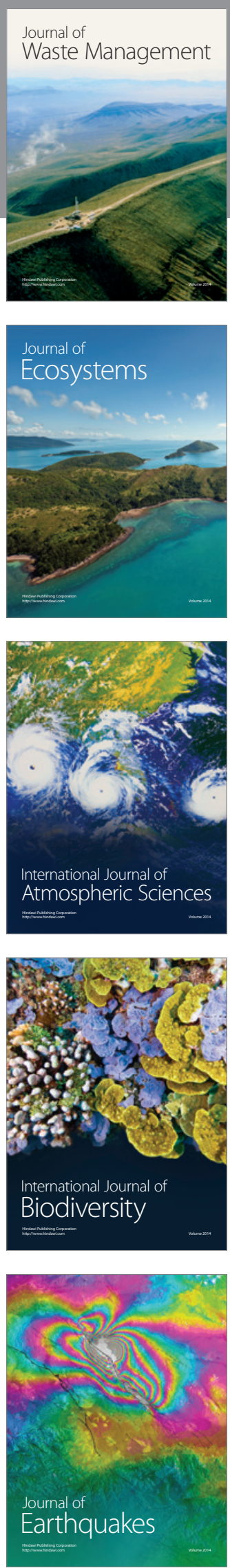
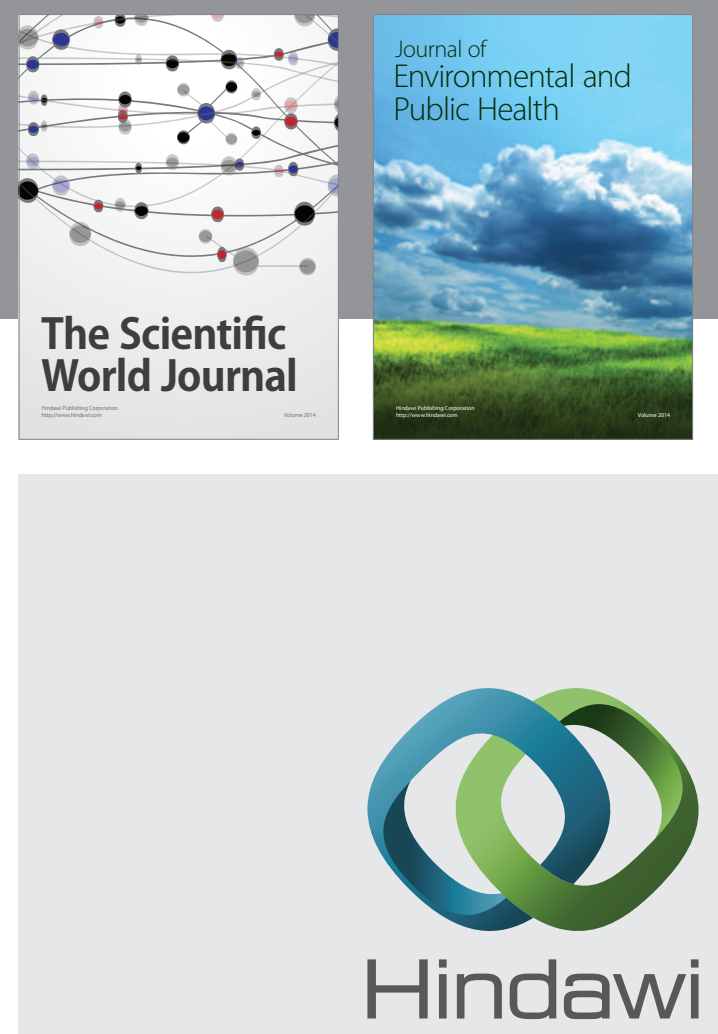

Submit your manuscripts at

http://www.hindawi.com
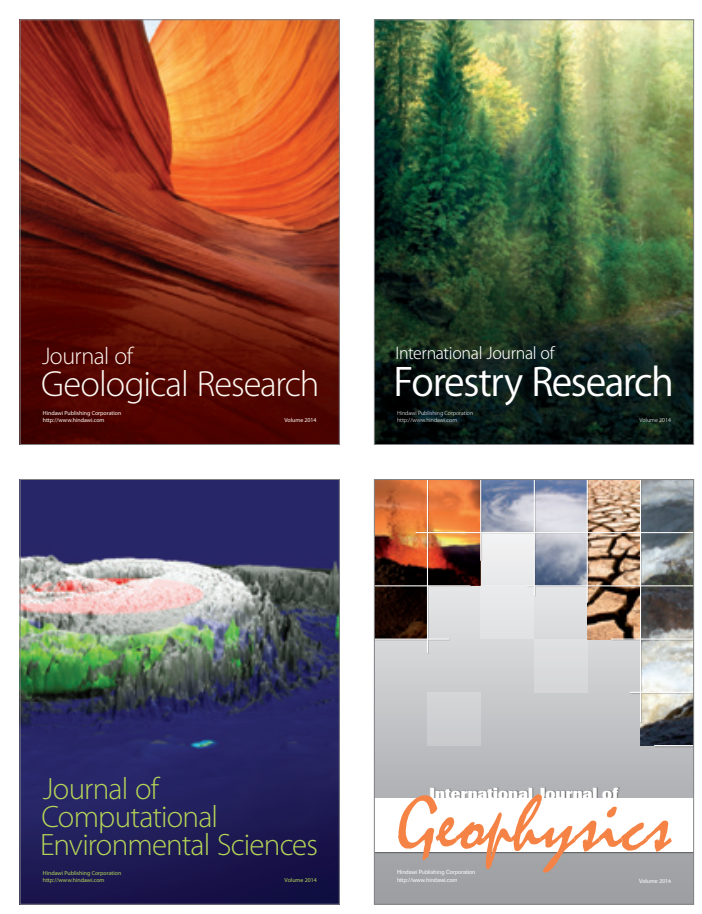
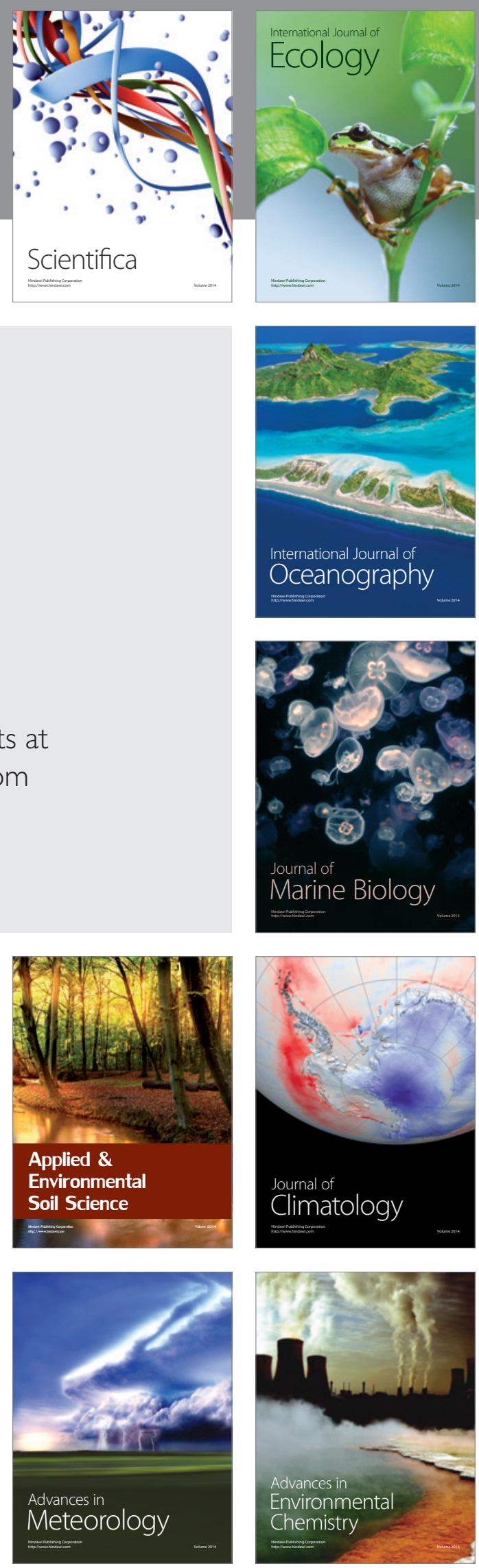